\title{
Analyses of energy metabolism and stress defence provide insights into Campylobacter concisus growth and pathogenicity
}

\author{
Melissa Yeow ${ }^{1}$, Fang Liu ${ }^{1}$, Rena Ma', Timothy J. Williams', Stephen M. Riordan² and Li Zhang ${ }^{1 *}$ (D)
}

\begin{abstract}
Campylobacter concisus is an emerging enteric pathogen that is associated with inflammatory bowel disease. Previous studies demonstrated that $\mathrm{C}$. concisus is non-saccharolytic and hydrogen gas $\left(\mathrm{H}_{2}\right)$ is a critical factor for $\mathrm{C}$. concisus growth. In order to understand the molecular basis of the non-saccharolytic and $\mathrm{H}_{2}$-dependent nature of $\mathrm{C}$. concisus growth, in this study we examined the pathways involving energy metabolism and oxidative stress defence in $C$. concisus. Bioinformatic analysis of C. concisus genomes in comparison with the well-studied enteric pathogen Campylobacter jejuni was performed. This study found that C. concisus lacks a number of key enzymes in glycolysis, including glucokinase and phosphofructokinase, and the oxidative pentose phosphate pathway. C. concisus has an incomplete tricarboxylic acid cycle, with no identifiable succinyl-CoA synthase or fumarate hydratase. C. concisus was inferred to use fewer amino acids and have fewer candidate substrates as electron donors and acceptors compared to C. jejuni. The addition of DMSO or fumarate to media resulted in significantly increased growth of $C$. concisus in the presence of $\mathrm{H}_{2}$ as an electron donor, demonstrating that both can be used as electron acceptors. Catalase, an essential enzyme for oxidative stress defence in C. jejuni, and various nitrosative stress enzymes, were not found in the $C$. concisus genome. Overall, C. concisus is inferred to have a non-saccharolytic metabolism in which $\mathrm{H}_{2}$ is central to energy conservation, and a narrow selection of carboxylic acids and amino acids can be utilised as organic substrates. In conclusion, this study provides a molecular basis for the non-saccharolytic and hydrogen-dependent nature of $C$. concisus energy metabolism pathways, which provides insights into the growth requirements and pathogenicity of this species.
\end{abstract}

Keywords: Campylobacter concisus, Campylobacter jejuni, Campylobacter, Hydrogen, Energy, Metabolism, Respiration

\section{Introduction}

Campylobacter species are fastidious Gram-negative, curved rod-shaped bacteria which require microaerobic to anaerobic conditions for growth [1]. Currently, the genus Campylobacter contains 40 species and subspecies [2]. While most Campylobacter species reside in the gastrointestinal tract of various animals as commensal bacterial species, some use humans as their natural host [2].

*Correspondence: L.Zhang@unsw.edu.au

${ }^{1}$ School of Biotechnology and Biomolecular Sciences, University of New South Wales, Kensington, Sydney 2052, Australia

Full list of author information is available at the end of the article
Campylobacter jejuni colonises the avian gut commensally, but it is a human pathogen causing gastroenteritis in both developing and developed countries [3-5], due to consumption of undercooked or contaminated chicken and other meat products [6-8]. C. jejuni contains two subspecies, C. jejuni subsp. jejuni and C. jejuni subsp. doylei [9]. Most of the cases of campylobacteriosis are caused by C. jejuni subsp. jejuni [10].

Campylobacter concisus is a human hosted Campylobacter species that colonizes the oral cavity of healthy individuals [11]. It is also an emerging enteric pathogen that has been associated with the development of 
inflammatory bowel disease (IBD) and other gastrointestinal diseases [12-19].

Campylobacter species have been historically considered to be non-saccharolytic, given that they have an incomplete Embden-Meyerhof-Parnas (EMP) glycolytic pathway that lacks hexose catabolism enzymes such as glucokinase and phosphofructokinase [20]. However, subsequent studies have amended this view. C. jejuni subsp. doylei 269.97 encodes a complete Entner-Doudoroff (ED) pathway, putatively acquired from Helicobacter, which suggests the potential to catabolise glucose [21]. The ED pathway has also recently been found in certain strains of the zoonotic enteric pathogen Campylobacter coli [22]. In addition, $C$. jejuni subsp. jejuni strains that have the genomic island (cj0480-cj0490) were demonstrated to have the ability to utilise fucose for growth, which is catabolized to pyruvate and lactate [23, 24]. The lower portion of the EMP is encoded in C. jejuni, and inferred to be involved in gluconeogenesis, which is dependent on anaplerotic enzymes to link it to the tricarboxylic acid (TCA) cycle [25].

As most strains of C. jejuni cannot utilise sugars [24, 26, 27], C. jejuni mainly relies on amino acids and C4-dicarboxylates as a primary energy source [28]. C. jejuni is known to preferentially use the amino acids aspartate, serine, glutamate, asparagine and proline [29-32], as well as the C4-dicarboxylates fumarate, succinate and malate [30]. Use of amino acids and C4-dicarboxylates for energy metabolism are interconnected as amino acids can be converted into C4-dicarboxylates that can enter the TCA cycle or be used in the electron transport chain for energy metabolism. Additionally, the C4-dicarboxylate transporters DcuA and DcuB found in C. jejuni are known to transport both fumarate and the dicarboxylic amino acid aspartate $[28,33]$.

C. jejuni has a highly branched electron transport chain, with a range of electron transport routes available, and the ability to use diverse substrates as electron donors [25,34]. This includes organic acids such as formate [35, 36], gluconate [37, 38], lactate [39], and in particular the TCA cycle intermediates pyruvate [40], 2-oxoglutarate [41], succinate [42], fumarate [42] and malate [36]. Hydrogen gas $\left(\mathrm{H}_{2}\right)$ [35, 43] and sulphite [44] can also be used as electron donors. In addition to oxygen-dependent respiration [45], a variety of alternative electron acceptors can be used by $C$. jejuni including fumarate [42, 46], nitrate [47], nitrite [48], trimethylamine $\mathrm{N}$-oxide (TMAO), dimethyl sulphoxide (DMSO) [49], and tetrathionate [50].

The molecular basis of the non-saccharolytic and hydrogen-dependent nature of $C$. concisus has not been previously made clear, because few studies have examined the genes and pathways involved in respiration and energy metabolism in $C$. concisus [51, 52]. Recently, more than 200 C. concisus genomes including three complete genomes have become available in public databases, providing the opportunity for us to investigate these pathways using bioinformatics analysis. This study investigates the interlinked pathways of central carbon metabolism, the electron transport chain, amino acid use, and oxidative stress defence mechanisms as previous studies on these pathways in C. jejuni have shown their importance for growth, motility, survival of the host immune response, and host colonization [25, 34, 42, 43, 53, 54].

\section{Results}

\section{C. concisus has an incomplete glycolytic pathway} and an incomplete pentose phosphate (PP) pathway

The EMP pathway of $C$. concisus is incomplete, lacking the genes for glucokinase $(g l k)$ and phosphofructokinase (pfk) (Fig. 1 and Additional file 1: Table S1), but possessing the gene for phosphoglucose isomerase (pgi) and all other genes, including those required for gluconeogenesis. A complete gluconeogenesis pathway is present in C. concisus: pyruvate carboxylase ( $p y c A B)$, phosphoenolpyruvate carboxykinase $(p c k A)$, and fructose 1,6-bisphosphatase $(f b p)$ have been identified (Fig. 1 and Additional file 1: Table S1). The PP pathway in $C$. concisus is incomplete; genes pertaining to the oxidative phase were not identified: glucose 6-phosphate dehydrogenase $(g d h)$ and 6-phosphogluconate dehydrogenase (zwf) (Fig. 1 and Additional file 1: Table S1). None of the genes of the ED pathway were identified in C. concisus (Figs. 1, 2, Additional file 1: Table S1 and Additional file 2: Table S2). Thus, C. concisus is unable to catabolise glucose to pyruvate through the EMP, PP or ED pathways. $C$. concisus lacks homologs of $C$. jejuni genes implicated in the catabolism of fucose (cj0481, cj0484, cj0485, cj0486, cj0487). Genes for the conversion of pyruvate to acetate via acetyl-CoA and acetylphosphate were identified in $C$. concisus: pyruvate-flavodoxin oxidoreductase (por), acetate kinase ( $a c k A)$, and phosphate acetyltransferase ( $p t a)$. (Additional file 1: Table S1). A $\mathrm{H}_{2}$-generating [NiFe] hydrogenase (Group 4a) was identified in the genome, for the disposal of reductant as $\mathrm{H}_{2}$.

\section{C. concisus has an incomplete TCA cycle}

Most of the genes in the TCA cycle were identified in C. concisus except for succinyl-CoA synthetase (sucCD) and fumarate hydratase (fumC). A C4-dicarboxylate transporter $(d c t A)$ is encoded for the uptake of the TCA cycle intermediate succinate. Other C4-dicarboxylate transporters $(d c u A, d c u B)$ are encoded for the uptake of the TCA cycle intermediate fumarate and aspartate. The genes required for the glyoxylate bypass were not identified in C. concisus: isocitrate lyase (aceA) 


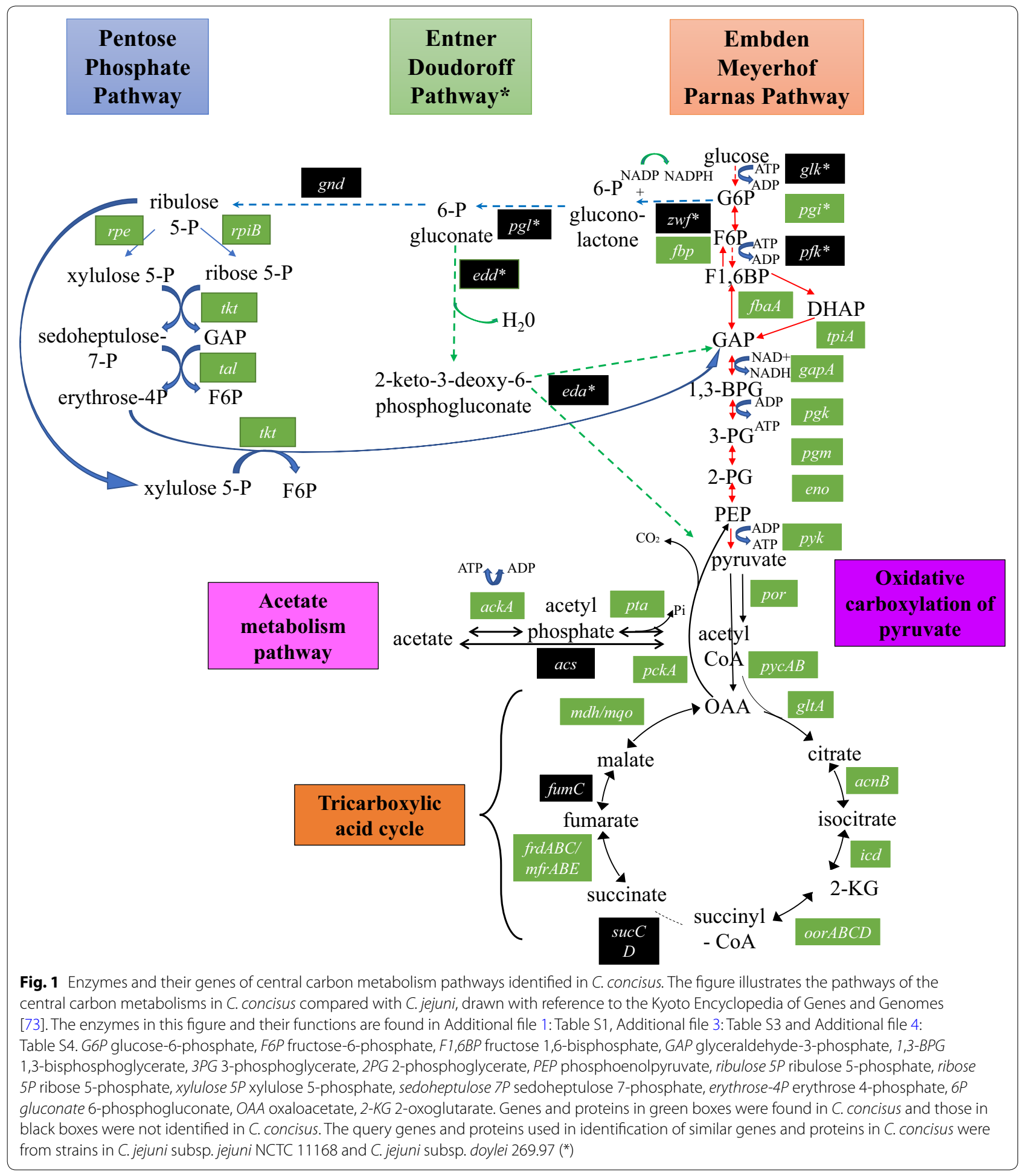

and malate synthase $(a c e B)$ (Fig. 1, Additional file 1: Table S1, Additional file 3: Table S3 and Additional file 4: Table S4).

\section{C. concisus may be able to use fewer electron donors than C. jejuni}

Genes for lactate oxidase (lutCBA) and lactate dehydrogenase (ldh) could not be identified in C. concisus, thus precluding use of the two pathways for use of lactate as an 


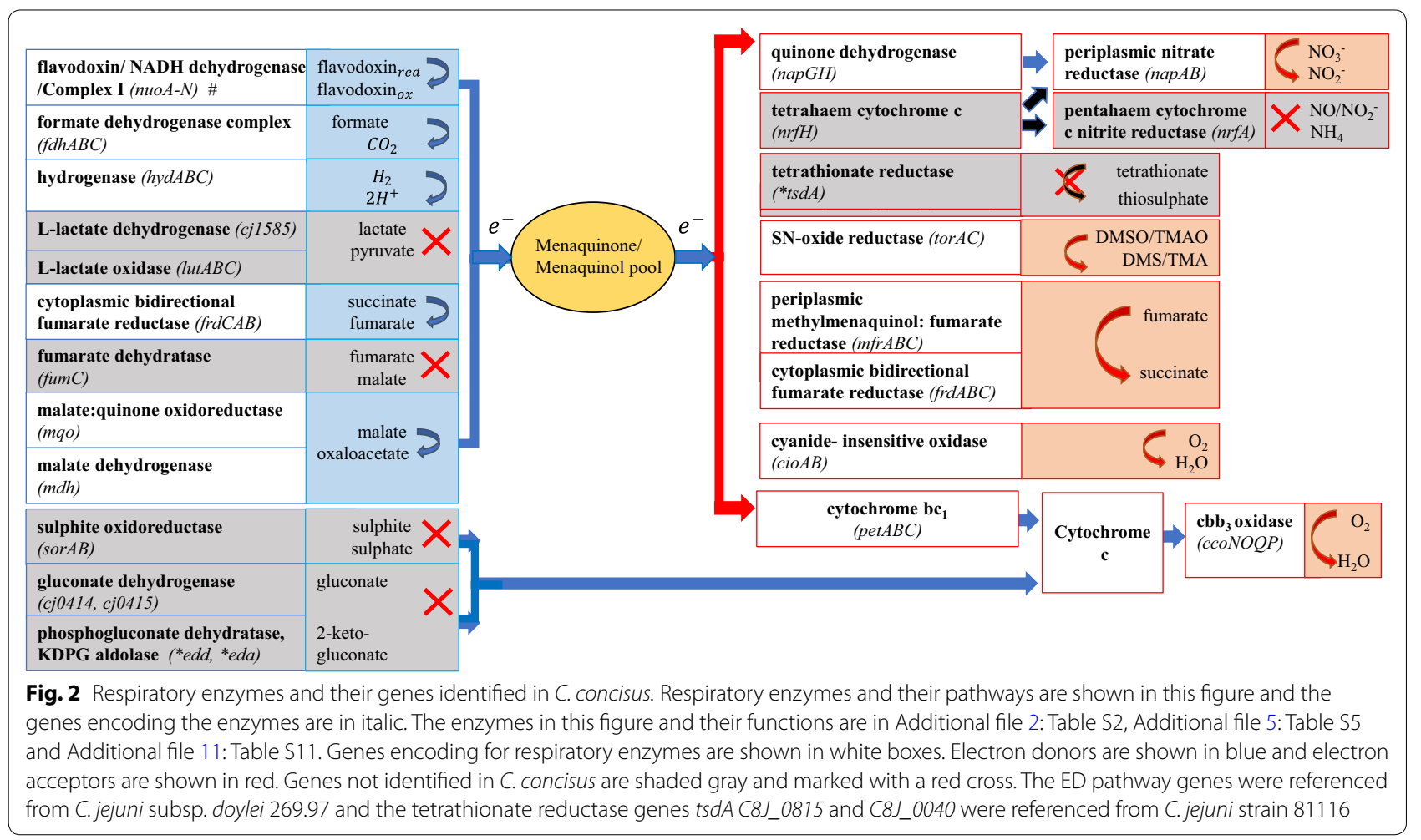

electron donor (Fig. 2 and Additional file 2: Table S2). C. concisus also lacks identifiable genes for gluconate dehydrogenase ( $g n d A B)$. Given the absence of the ED pathway (see above) C. concisus is unable to catabolise gluconate to pyruvate (Fig. 2 and Additional file 1: Table S1 and Additional file 2: Table S2).

However, genes encoding enzymes for the oxidation of other electron donors were identified in C. concisus, such as malate:quinone oxidoreductase ( $m q o$ ), pyruvate:flavodoxin oxidoreductase (por), 2-oxoglutarate:acceptor oxidoreductase (oor $A B C D)$, formate dehydrogenase (fdhABC), succinate dehydrogenase ( $\mathrm{rd} A B C$ ), and $\mathrm{H}_{2}$-uptake [NiFe] hydrogenase (hydABCD) (Group 1b) (Fig. 2 and Additional file 2: Table S2).

\section{C. concisus may be able to use fewer electron acceptors than C. jejuni}

There are two pathways in C. concisus for use of oxygen as an electron acceptor, via cytochrome $\mathrm{C}$ oxidase (encoded by $c c o N O Q P)$, or cyanide-insensitive quinol oxidase $($ cio $A B)$. Genes encoding nitrite reductase $(n r f A H)$ were not identified in $C$. concisus, suggesting an inability to use nitrite as an electron acceptor. Also, homologs for the $C$. jejuni tetrathionate reductase $t s d A$ (C8J_0815) and a $t s d A$ paralog (C8J_0040) were not found in C. concisus (Fig. 2).

Genes encoding the periplasmic nitrate reductase (napABGH) which allows nitrate to be used as an electron acceptor, were found in C. concisus. Genes encoding the periplasmic methylmenaquinol fumarate reductase $(m f r A B E)$ as well as a dual-functioning cytoplasmic fumarate reductase $(\mathrm{frd} A B C)$ were found in C. concisus (Fig. 2 and Additional file 5: Table S5). Methylmenaquinol fumarate reductase (Mfr) and fumarate reductase (Frd) allow use of fumarate as an electron acceptor, reducing fumarate to succinate using electrons from the menaquinone pool. C. concisus encodes an anaerobic $\mathrm{C}_{4}$-dicarboxylate membrane transporter $(\mathrm{dcuB})$ for succinate efflux. TMAO/DMSO reductase (torAC) for use of $\mathrm{SN}$-oxides as electron acceptors were also found in $C$. concisus (Fig. 2 and Additional file 5: Table S5).

\section{C. concisus may be able to use fewer amino acids than $C$. jejuni}

Genes for the serine transporter $(s d a C)$, serine dehydratase $(s d a A)$ and proline dehydratase (putA) were not found in C. concisus, though the proline symporter (putP) was identified. Additionally, branched chain amino acids do not appear to be taken up by $C$. concisus, due to lack of the LIV transporter system (livJKHMGF). The pathogenesis associated glutamine $\mathrm{ABC}$ transporter permease (paqP) and ATPase (paqQ) as well as the $\mathrm{ABC}$ transporter system encoded by the peb locus which transports glutamate and aspartate were identified in C. concisus. Glutamate dehydrogenase ( $g h d A$ ) and aspartate:glutamate 
transaminase $(a s p B)$ were also encoded, which suggests that $C$. concisus can convert glutamate to 2-oxoglutarate and ammonia, or generate aspartate and 2-oxoglutarate from glutamate and oxaloacetate via transamination. Aspartate ammonia lyase ( $\operatorname{ssp} A$ ) was also encoded, for conversion of aspartate to fumarate and ammonia. Periplasmic asparaginase (ans $B$ ) was also found, allowing use of asparagine as a source of aspartate (Fig. 3, Additional file 6: Table S6 and Additional file 7: Table S7).

\section{C. concisus has fewer enzymes to deal with oxidative and nitrosative stress than $C$. jejuni}

The katA gene, which encodes catalase that detoxifies hydrogen peroxide, was not found in $C$. concisus. Ten other genes that are involved in dealing with oxidative stress in C. jejuni were found in C. concisus (Fig. 4). The three genes that encode enzymes to deal with nitrosative stress in C. jejuni (cgb, $c t b, n r f a)$, were not found in $C$. concisus.

\section{Without the presence of $\mathrm{H}_{2}$, sodium fumarate did not increase $C$. concisus growth}

The effects of sodium fumarate on $C$. concisus growth under anaerobic conditions with and without the presence of $\mathrm{H}_{2}$ were examined. Under anaerobic conditions with $\mathrm{H}_{2}$, all three strains cultured on horse blood agar (HBA) plates and HBA plates supplemented with 0.05 , 0.2 and $0.4 \%(\mathrm{w} / \mathrm{v})$ sodium fumarate showed an increase in colony forming unit (CFU) compared to the initial inoculum, with fold changes greater than one (Fig. 5). Compared to the same strains grown on HBA plates, all

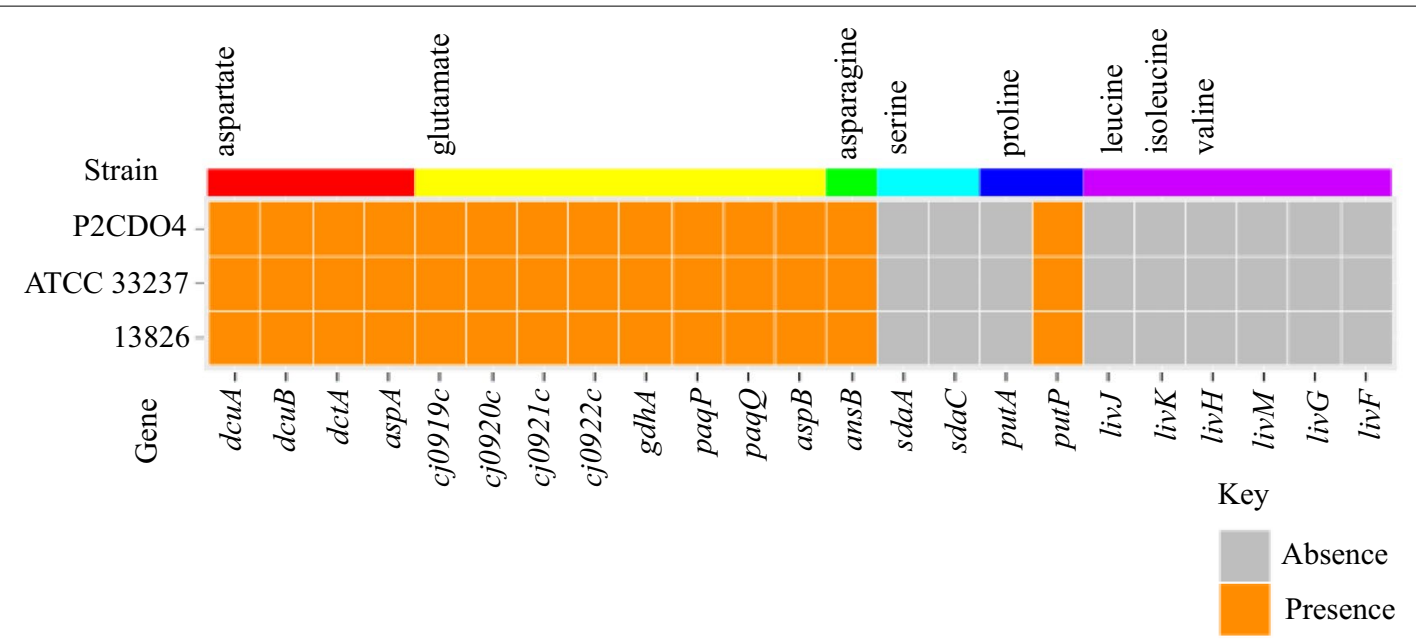

Fig. 3 Genes for enzymes of amino acid use pathways identified in C. concisus. The genes for the enzymes of amino acid use pathways identified in C. concisus are shown in this figure. The corresponding amino acid used by the enzymes is shown by the colour coded bar above. The enzymes in this figure and their functions are in Additional file 6: Table S6 and Additional file 7: Table S7

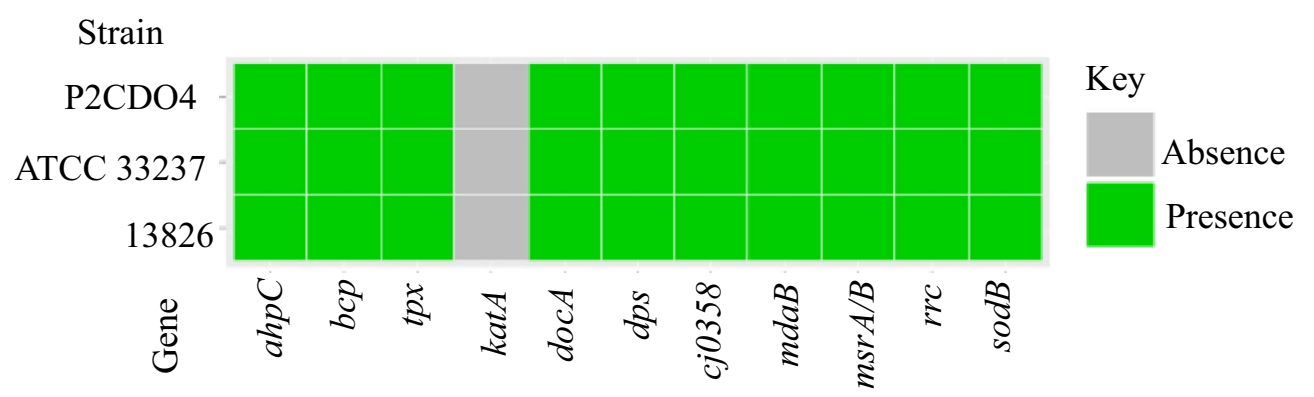

Fig. 4 Genes for enzymes of oxidative stress defence pathways identified in C. concisus. The genes for the enzymes of oxidative stress defence pathways identified in C. concisus are shown in this figure. The enzymes in this figure and their functions are in Additional file 8: Table S8 and Additional file 9: Table S9 

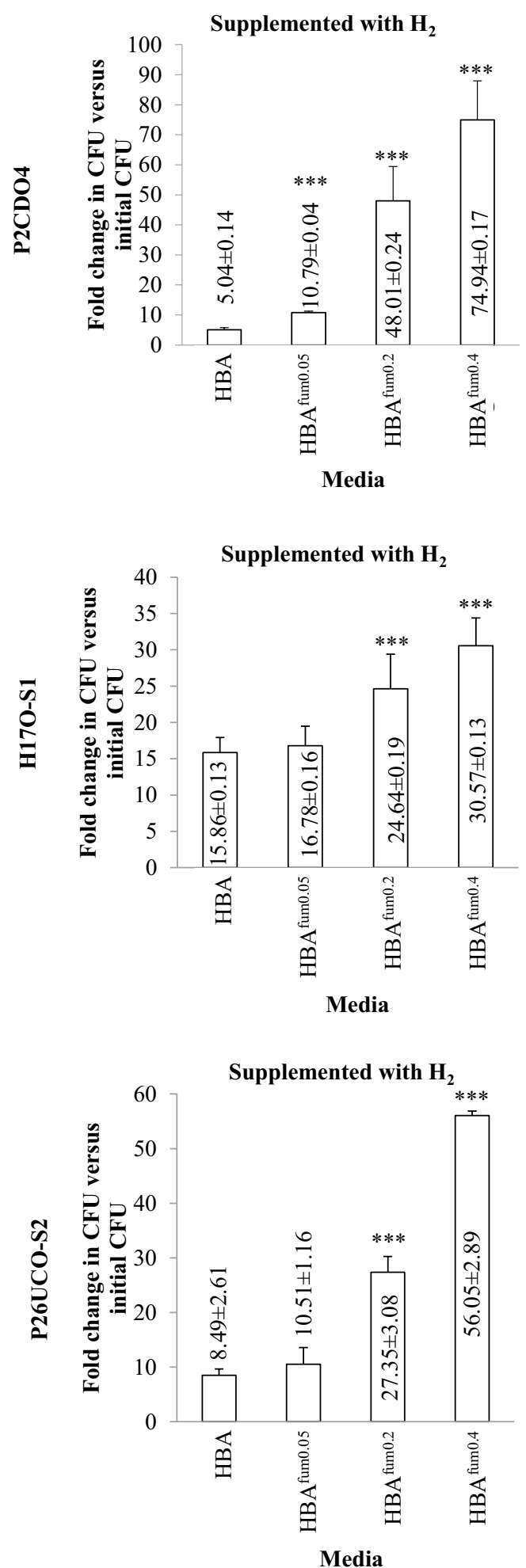

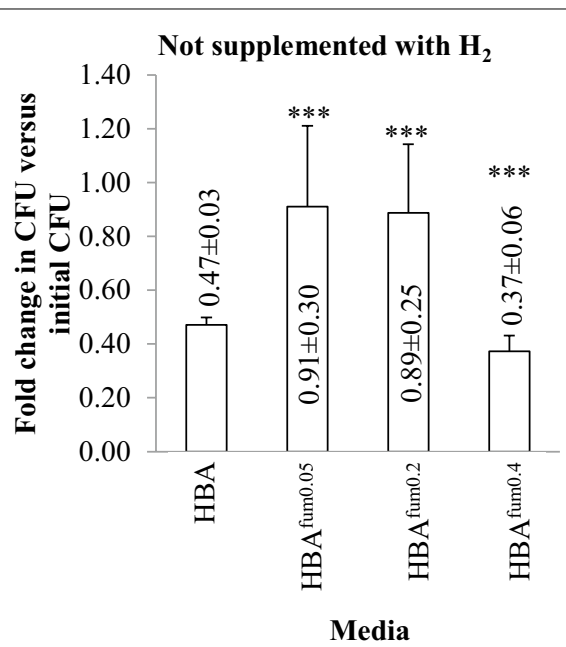

Not supplemented with $\mathrm{H}_{2}$

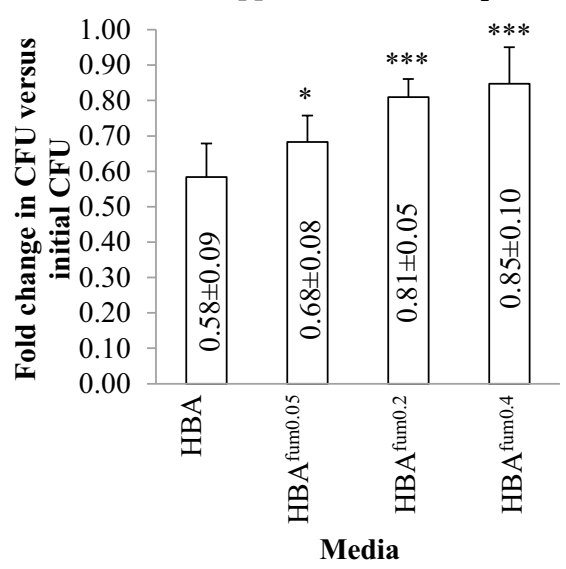

Not supplemented with $\mathrm{H}_{2}$

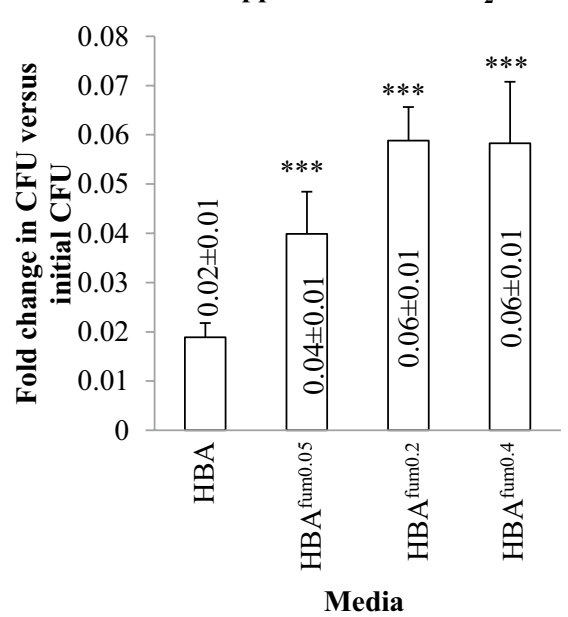

Fig. 5 The effects of sodium fumarate on C. concisus growth under anaerobic conditions with and without $\mathrm{H}_{2}$ gas. C. concisus strains P2CDO4, $\mathrm{H} 17 \mathrm{O}-\mathrm{S} 1$, and P26UCO-S2 were cultured on two sets of HBA, HBA ${ }^{\text {fum } 0.05}, \mathrm{HBA}^{\text {fum0.2 }}$ and $\mathrm{HBA}^{\text {fum } 0.4}$. One set was incubated in anaerobic conditions with hydrogen and the other was incubated without hydrogen. Both the starting number of bacteria and total after incubation was enumerated for all strains. Fold changes were calculated relative to the starting number of bacteria from quadruplicate counts. In anaerobic conditions with hydrogen, all strains showed increased growth, with the greatest increase on HBA fum0.4. In anaerobic conditions without hydrogen, all strains had a CFU lower than the initial inoculation CFU. * Indicates statistical significance compared to CFU on HBA in the same condition. ${ }^{* *} P<0.001$ 
three strains grown on HBA plates supplemented with 0.2 and $0.4 \%$ sodium fumarate had a significantly higher CFU $(P<0.05)$. Strain P2CDO4 also showed a significantly higher growth on HBA plates supplemented with $0.05 \%$ sodium fumarate as compared to the same strain grown on HBA plates $(P<0.05$, Fig. 5$)$.

In conditions without $\mathrm{H}_{2}$, all three $C$. concisus strains did not grow; their CFU fold changes cultured on HBA plates and HBA plates supplemented with different concentrations of sodium fumarate were all below one as compared to the initial inoculum CFU. Although the decrease in C. concisus CFU on HBA plates containing $0.05-0.04 \%$ was less than that in HBA plates without sodium fumarate, the overall results show that sodium fumarate did not increase $C$. concisus growth without the presence of $\mathrm{H}_{2}$ (Fig. 5).

\section{DMSO increased the growth of $C$. concisus}

In order to examine the effects of DMSO on C. concisus growth, $C$. concisus strains were cultured on HBA plates containing $0.2 \%$ of DMSO ( $\left.\mathrm{HBA}^{\mathrm{DMSO}}\right)$. DMSO significantly enhanced the growth of all three $C$. concisus strains being tested (Fig. 6). The levels of growth of $C$. concisus strains P2CDO4, H17O-S1, and P26UCO-S2 on $\mathrm{HBA}^{\mathrm{DMSO}}$ plates were $4 \pm 0.4,3 \pm 0.4$ and $18 \pm 1.1$ fold of those on HBA plates respectively, in which the increases were statistically significant $(P<0.001, P<0.01$, and $P<0.001$ respectively).

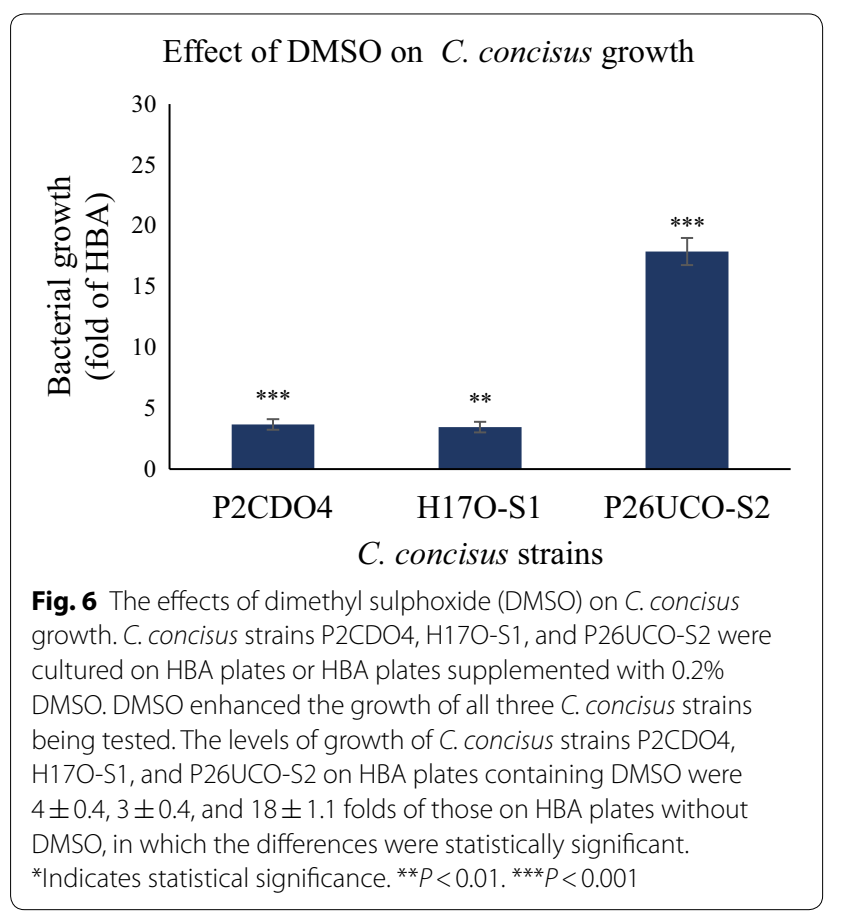

\section{Discussion}

In this study, we examined the pathways of energy metabolism and stress defence in C. concisus by analysis of the presence of relevant genes in C. concisus genomes. We also examined whether sodium fumarate increased $C$. concisus growth without the presence of $\mathrm{H}_{2}$ and whether DMSO affects the growth of $C$. concisus.

We found that $C$. concisus does not have complete metabolic pathways for the utilization of glucose [55]. The lack of $g l k$ and $p f k$ genes indicates that $C$. concisus is unable to convert glucose to fructose-1,6-bisphosphate using the EMP pathway. The lack of $g d h$ and $z w f$ shows that $C$. concisus is unable to metabolise glucose via the PP pathway (Fig. 1). Furthermore, C. concisus does not have genes for the ED pathway or for fucose catabolism, unlike as documented in certain C. jejuni strains (Fig. 1 and Additional file 1: Table S1 and Additional file 5: Table S5) [38]. Collectively, these findings support the previous characterization of C. concisus as non-saccharolytic [1].

We found that $C$. concisus has an incomplete TCA cycle (Fig. 2, Additional file 1: Table S1). Although C. concisus encodes most of the enzymes in the TCA cycle, it lacks both fumC and sucCD; the former is required for the production of fumarate from malate, the latter is necessary for generation of succinate from succinyl-CoA. It has previously been reported that succinate is an end product of $C$. concisus growth, generated from fumarate reduction [55]. The two enzymes Frd or Mfr are available in C. concisus to reduce fumarate (Fig. 2). During oxygenindependent respiration, fumarate reduction in $C$. jejuni generates succinate that is secreted during growth [28], and a DcuB transporter is encoded for succinate efflux.

C. concisus has the capacity to produce most TCA cycle intermediates, which donate reducing equivalents to the respiratory chain for energy metabolism (Fig. 2, Additional file 1: Table S1). Although a fumarate hydratase was initially annotated in C. concisus, the genes are more likely to encode tartrate dehydratase based on sequence identity [56]. C. concisus encodes aspartate ammonialyase $(\operatorname{asp} A)$; aspartate is a likely source of fumarate in $C$. concisus, in addition to fumarate directly sourced from the host diet [57]. The genome encodes transporters for the uptake of both aspartate and fumarate. The inability to use proline and serine by C. concisus (Fig. 3, Additional file 6: Table S6 and Additional file 7: Table S7) is interesting as proline utilization is considered important for $C$. jejuni intestinal colonization of mice [58], and serine utilization is critical for $C$. jejuni host colonization in chicks and mice $[58,59]$.

The genes necessary for pyruvate conversion to acetate (por, pta, ackA) were found in C. concisus; thus we infer the potential to generate ATP via substrate-level 
phosphorylation. It has been found previously that $C$. concisus generated acetate, although strain variation was noted by that study which detected acetate in three out of six C. concisus strains they examined [55].

Our findings also provide an explanation at the genomic level for $C$. concisus growth requirements. $C$. jejuni has genes encoding enzymes for all reactions in the TCA cycle; thus it is able to generate reducing equivalents for ATP production via the electron transport chain. In contrast, $C$. concisus is unable to use succinyl$\mathrm{CoA}$ to generate succinate, fumarate, malate, $\mathrm{FADH}_{2}$ and NADH molecules in the TCA cycle due to the lack of sucCD genes and cannot generate fumarate from malate due to lack of fumC (Fig. 1).

However, C. concisus has genes encoding enzymes for utilizing the amino acids glutamate, aspartate and asparagine (Fig. 3), as well as several electron donors, such as $\mathrm{H}_{2}$, formate, succinate and malate (Fig. 2). Given this, supplementation of these amino acids and electron donors may potentially increase the growth of $C$. concisus and the effects of some of these substances on C. concisus growth were examined previously and in this study.

$\mathrm{H}_{2}$ gas has been demonstrated to be a highly effective electron donor for $C$. concisus growth [51, 60]. It has been previously demonstrated that $C$. concisus had very low growth under anaerobic conditions and no growth under microaerobic conditions without $\mathrm{H}_{2}$ [42]. In the presence of $\mathrm{H}_{2}$, C. concisus was able to grow under microaerobic conditions and the growth under anaerobic conditions greatly increased [42]. C. concisus has both a $\mathrm{H}_{2}$-uptake hydrogenase (Hyd) and a $\mathrm{H}_{2}$-generating hydrogenase (Hyf) [51]. Site-directed mutagenesis demonstrated the critical role of $\mathrm{H}_{2}$ oxidation for C. concisus growth, with C. concisus additionally found to exhibit extremely high $\mathrm{H}_{2}$-uptake hydrogenase activity [51]. Although $\mathrm{H}_{2}$ evolution has been detected for $C$. concisus [55], the level of $\mathrm{H}_{2}$ produced by $C$. concisus produced only poor growth of C. concisus [60]. Thus, uptake of exogenous $\mathrm{H}_{2}$ generated by other bacterial species is a critical factor affecting the growth of C. concisus in the human gastrointestinal tract.

Fumarate can be used as an electron acceptor, with genes encoding a periplasmic Mfr and a bifunctional cytoplasmic Frd present in C. concisus (Fig. 2). However, as with the periplasmic nitrate reductase (Nap), Frd cannot contribute to generation of the membrane potential, and functions solely as an electron sink [33]. Sodium fumarate has been previously demonstrated to greatly increase the growth of $C$. concisus in the presence of $\mathrm{H}_{2}$ [61], which in combination with our study, showed that fumarate is an effective electron acceptor in C. concisus. This is further supported by proteomic data which show that fumarate reductase proteins (WP_012001794.1，WP_012001792.1，WP_012001793.1) had increased abundance in response to addition of fumarate [61].

Formate is also a potential electron donor in C. concisus, which encodes the formate dehydrogenase complex (fdhABC) (Fig. 2). Further, formate oxidation might be coupled to a $\mathrm{H}_{2}$-generating hydrogenase in a formate hydrogen lyase complex as in Escherichia coli [62]. However, previous studies found that supplementation of media with formate did not support $C$. concisus growth. C. concisus growth was inhibited by $0.2 \%$ formate in anaerobic conditions with $5 \%$ hydrogen [61]; growth did not occur in $20 \mathrm{mM}$ formate supplemented media under microaerobic conditions ( $5 \%$ oxygen) without hydrogen; and poor growth was observed in $20 \mathrm{mM}$ formate supplemented media under anaerobic conditions without hydrogen [51]. Further investigation into the effect of different concentrations of formate on C. concisus is warranted to shed light on this paradoxical phenomenon.

This study found that $C$. concisus has genes encoding enzymes for using nitrate, DMSO/TMAO, fumarate, and $\mathrm{O}_{2}$ as electron acceptors (Fig. 2). As mentioned above, fumarate is an effective electron acceptor in C. concisus [61]. The good growth of $C$. concisus under anaerobic conditions in the presence of $\mathrm{H}_{2}$ suggests that nitrate present in media may be used as an electron acceptor. In this study, we showed that DMSO increased the growth of $C$. concisus in the presence of $\mathrm{H}_{2}$, showing that it can be used effectively as an electron acceptor in C. concisus (Fig. 6). While nitrite reductase genes $n r f C D$ have been previously reported in C. concisus [51], it is unlikely to be functional due to lack of a gene for the catalytic subunit NrfA [48]. C. concisus also has the potential to use $\mathrm{O}_{2}$ as a terminal electron acceptor as it has the genes encoding a cyanide-insensitive oxidase $(\operatorname{cio} A B)$ and ubiquinolcytochrome c oxidoreductase (ccoNOQP), also referred to as the cytochrome bc ${ }_{1}$ complex (Fig. 2). However, the abilities of $C$. concisus using $\mathrm{O}_{2}$ as an electron acceptor may have been limited due to its lack of catalase, which was found to be essential for hydrogen peroxide resistance in C. jejuni [53].

Catalase catalyzes the decomposition of hydrogen peroxide, a highly reactive product generated during using molecular oxygen as the final electron acceptor. C. concisus does not have katA (Fig. 4, Additional file 8: Table S8 and Additional file 9: Table S9) and was previously demonstrated to be catalase negative [55]. C. concisus has the gene encoding superoxide dismutase, which was found to be expressed in C. concisus when fumarate was added to media [61]. The overall ability of $C$. concisus in dealing with reactive oxygen stress appears lower compared to $C$. jejuni, which has both catalase and superoxide dismutase $[53,63]$. This is especially likely as it has been shown that catalase is essential for $C$. jejuni 
in vitro hydrogen peroxide resistance, and intramacrophage survival via katA mutant experiments [53]. This may explain a previous observation that $C$. concisus grew better under anaerobic than microaerobic conditions in the presence of $5 \% \mathrm{H}_{2}[60]$. C. concisus has been demonstrated to grow better in microaerobic conditions than in anaerobic conditions when $\mathrm{H}_{2}$ concentration was raised to $20 \%$ [51]. This interesting observation that a higher level of $\mathrm{H}_{2}$ appeared to increase the ability of C. concisus to resist oxidative stress may be due to antioxidant effects of hydrogen via reduction of hydroxyl radicals and peroxynitrite [64].

C. concisus does not have any of the three known nitrosative stress defence enzymes used by $C$. jejuni. However, it was previously reported that C. concisus strain 13826 has a nitric oxide reductase NorZ and a nitrous oxide reductase NosZ [51], which are not present in C. jejuni [65]. This study found that these enzymes were present in all three fully sequenced strains of $C$. concisus. This may explain the increased growth of $C$. concisus strain ATCC 33237 in response to an increased concentration of nitric oxide donor sodium nitroprusside [66].

Whereas we found that $C$. concisus lacked the gene that encodes tetrathionate reductase in C. jejuni strain 81116, a tetrathionate reductase gene has been reported in $C$. concisus strain 13826 that is similar to the tetrathionate reductase $(t t r)$ in Salmonella typhimurium [51]. The latter study also demonstrated a functional tetrathionate reduction pathway using site-directed mutagenesis, and found that addition of tetrathionate to growth media resulted in increased growth of C. concisus [51].

Findings from this study and previous studies suggest that the pathogenicity of $C$. concisus is not only determined by the virulence of individual $C$. concisus strains but also the microenvironment of the gastrointestinal tract of individual hosts particularly the availability of $\mathrm{H}_{2}$ for growth. As the composition of microbiota and diet are the two major factors influencing the production of $\mathrm{H}_{2}$ in the gastrointestinal tract, their impact on C. concisus enteric pathogenicity warrants future investigation.

\section{Conclusions}

This study found that $C$. concisus lacks critical genes in the central carbon metabolism pathways and has an incomplete TCA cycle, with a missing succinyl-CoA synthetase $(\operatorname{sucCD})$ and fumarate hydratase $($ fumC). This study showed that fumarate and DMSO are effective electron acceptors in C. concisus in the presence of $\mathrm{H}_{2}$ as an electron donor. C. concisus was also found to have fewer genes that encode enzymes for utilizing amino acids, electron donors and acceptors, as well as stress defence compared to C. jejuni, although this study cannot rule out the possibility that $C$. concisus may use other alternative pathways. In conclusion, this study provides a molecular basis for the non-saccharolytic and hydrogendependent nature of $C$. concisus via its energy metabolism and stress defence pathways, which provides insights into the growth requirements and pathogenicity of this species.

\section{Materials and methods}

Bioinformatic methods used to examine the presence of genes encoding enzymes in energy production and stress defence in $C$. concisus

The sequences of the reference genes from reference bacterial strains (see the section below) were obtained from the National Center for Biotechnology Information (NCBI) database. Proteins encoded by these genes were used as query sequences to identify similar proteins in the three $C$. concisus strains with fully sequenced genomes (13826, ATCC 33237 [67] and P2CDO4 [15]) using the BLASTp program [68]. Previously published criteria (more than $30 \%$ identity, E-values $<10^{-10}$ and bit scores of $>50$ ) were used to determine presence of a gene [69]. Protein sequences that were identified as hydrogenases based on catalytic domains were classified further using the hydrogenase classifier HydDB [70].

The nucleotide sequences of the reference genes were also used as query genes to identify similar nucleotide sequences in the genomes of all 249 C. concisus strains in the public database including the three fully sequenced genomes using BLASTn as further verification of the data obtained from using the BLASTp comparison using the previously mentioned criteria [69]. The C. concisus strains used in the analysis of this study are in Additional file 10: Table S10.

\section{The reference genes used to identify similar genes and proteins in the $C$. concisus genomes}

To examine the presence of genes encoding enzymes in the pathways of energy production and stress defence in $C$. concisus, a total of 206 reference genes and their encoded proteins from E. coli and C. jejuni were used as query genes and proteins to identify similar genes and proteins in C. concisus genomes using the methods described above.

Reference genes and their encoded proteins from E. coli strain K-12 MG1655 (NC_000913.3), C. jejuni subsp. jejuni strain NCTC 11168 (NC_002163.1) and C. jejuni subsp. doylei strain 269.79 (NC_009707.1) were used as the query genes and proteins. The choice of $E$. coli strain K-12 MG1655 as a reference strain was due to its well-studied metabolic pathways, in particular its glycolytic pathways [71]. C. jejuni strain NCTC 11168 (NC_002163.1) was used as a reference because 
it is a member of the Campylobacter genus with wellstudied metabolic pathways [26, 72], except for the ED pathway, which was discovered in C. jejuni subsp. doylei 269.97 (NC_009707.1) and not found in most C. jejuni subsp. jejuni strains [38]. In addition, the tetrathionate reductase genes $t s d A$ (C8J_0815) and the $t s d A$ paralog (C8J_0040) were discovered in C. jejuni strain 81116 [50]. As such, $C$. jejuni strain 81116 was used as a reference for the tetrathionate reductase pathway.

The reference genes and encoding proteins that were used as query genes and proteins for identification of similar genes and enzymes in the central carbon metabolic pathways of $C$. concisus are listed in Additional file 3: Table S3 (E. coli strain K-12 reference genes) and Additional file 4: Table S4 (C. jejuni subsp. jejuni and $C$. jejuni subsp. doylei reference genes). The reference genes and proteins that were used as query genes or proteins for identification of similar respiratory chain enzymes in jejuniC. concisus are listed in Additional file 11: Table S11 (C. jejuni subsp. reference genes). The reference genes and encoded proteins from C. jejuni subsp. jejuni NCTC 11168, C. jejuni strain 81116 (livJKHMGF) and E. coli strain K-12 $(g d h A)$ were used as query genes and proteins for identification of enzymes involved in amino acid use in are listed in Additional file 7: Table S7. The reference genes and encoded proteins from $C$. jejuni subsp. jejuni NCTC 11168 were used as query genes and proteins for identification enzymes involved in oxidative and nitrosative stress in C. concisus are listed in Additional file 9: Table S9. All additional references cited in additional files can be found in Additional file 12.

\section{Quantitative analysis of $C$. concisus growth on media supplemented with sodium fumarate under anaerobic conditions with and without hydrogen}

We previously showed that sodium fumarate significantly increased the growth of $C$. concisus under anaerobic conditions in the presence of $\mathrm{H}_{2}$, supporting that fumarate is an electron acceptor in C. concisus [61]. To examine whether fumarate is an electron donor in C. concisus, in this study, we compared the growth of $C$. concisus under anaerobic conditions with and without $\mathrm{H}_{2}$ on media supplemented with sodium fumarate.

Three strains of $C$. concisus were randomly selected to examine their growth when sodium fumarate (SigmaAldrich, Missouri, USA) is supplemented in media and incubated in anaerobic conditions with and without hydrogen. These strains were P2CDO4, H17O-S1 and P26UCO-S; each are orally isolated C. concisus strains from a patient with Crohn's disease, a healthy control and a patient with ulcerative colitis, respectively. Each strain was first cultured on HBA (Oxoid, Hampshire, UK) with 6\% defibrinated horse blood and incubated in anaerobic conditions with $5 \%$ hydrogen as previously described [61]. Cultures were prepared to an optical density of 0.1 at a wavelength of $595 \mathrm{~nm}$ and $5 \mu \mathrm{L}$ was inoculated onto two sets of HBA, HBA with $0.05 \%$ sodium fumarate $\left(\mathrm{HBA}^{\text {fum0.05 }}\right)$, HBA with $0.2 \%$ sodium fumarate $\left(\mathrm{HBA}^{\mathrm{fum} 0.2}\right.$ ) and HBA with $0.4 \%$ sodium fumarate $\left(\mathrm{HBA}^{\text {fum } 0.4}\right)$. Each set of media was incubated in anaerobic conditions either with or without $5 \%$ hydrogen available. Plates were incubated for $48 \mathrm{~h}$ and CFU were subsequently quantified as previously described [61]. The CFU of the initial cell suspension used for inoculation was also quantified, allowing determination of $C$. concisus growth under cultivation conditions used [61]. Experiments were repeated three times.

\section{Examination of the effects of DMSO on the growth of $C$. concisus}

The above three $C$. concisus strains were also used to examine whether DMSO affects the growth of C. concisus. C. concisus strains were cultured as described above. Cultures were prepared to optical density of 0.025 at a wavelength of $595 \mathrm{~nm}$ and $5 \mu \mathrm{L}$ was inoculated onto two sets of HBA plates or HBA plates supplemented with $0.2 \%$ DMSO (ThermoFisher Scientific, Massachusetts, USA) for $48 \mathrm{~h}$ under anaerobic conditions containing 5\% $\mathrm{H}_{2}$. CFUs were determined as described previously [61].

\section{Statistical analysis}

The CFUs of $C$. concisus strains cultured on HBA plates supplemented with sodium fumarate and DMSO under different atmospheric conditions were compared using 2 -tailed $t$-tests. A $P$ value of less than 0.05 was considered significant.

\section{Supplementary information}

Supplementary information accompanies this paper at https://doi. org/10.1186/s13099-020-00349-6.

Additional file 1: Table S1. NCBI locus tags for genes involved in central carbon metabolism.

Additional file 2: Table S2. NCBI locus tags for genes involved in use of electron donors of $C$. concisus

Additional file 3: Table S3. Query genes and proteins from E. coli strain K-12 MG1655 for identification of genes and proteins in C. concisus central carbon metabolism pathways.

Additional file 4: Table S4. Query genes and proteins from C. jejuni subsp. jejuni NCTC 11168 for identification of genes and proteins of C. concisus central carbon metabolism pathways.

Additional file 5: Table S5. NCBI locus tags for genes involved in use of electron acceptors.

Additional file 6: Table S6. NCBI locus tags for genes involved in amino acid use.

Additional file 7: Table S7. Query genes and proteins from C. jejuni subsp. jejuni NCTC 11168, E. coli strain K-12 MG1655, and C. jejuni subsp. 
jejuni strain 81116 used to identify genes and proteins for amino acid use in C. concisus.

Additional file 8: Table S8. NCBI locus tags for genes involved in oxidative stress.

Additional file 9: Table S9. Query genes and proteins from C. jejuni subsp. jejuni NCTC 11168 that were used to identify genes and proteins for oxidative and nitrosative stress defence in $C$. concisus.

Additional file 10: Table S10. C. concisus strains used in BLASTn analysis.

Additional file 11: Table S11. Genes encoding electron donors and acceptors investigated in C. concisus as referenced from C. jejuni subsp. jejuni NCTC 11168 and C. jejuni subsp. jejuni strain 81116.

Additional file 12. Additional references cited in additional files 7,9 and 11.

\section{Authors' contributions}

MY: Conducted bioinformatics analysis, analyzed data and wrote manuscript. FL: Performed DMSO experiment, provided help with bioinformatic analysis and co-edited manuscript. RM: Performed fumarate experiment and co-edited manuscript. TJW: Provided guidance on bioinformation analysis, discussed data and co-edited manuscript. SMR: Conceived the project and co-edited manuscript. LZ: Conceived the project, discussed data and co-wrote manuscript. All authors read and approved the final manuscript.

\section{Funding}

This work is supported by the University of New South Wales Faculty Research Funding awarded to LZ (PS46772).

\section{Availability of data and materials}

All data generated or analysed during this study are included in this published article (and its additional information files).

\section{Ethics approval and consent to participate}

Not applicable.

\section{Consent for publication}

Not applicable.

\section{Competing interests}

The authors declare that they have no competing interests.

\section{Author details}

1 School of Biotechnology and Biomolecular Sciences, University of New South Wales, Kensington, Sydney 2052, Australia. ${ }^{2}$ Gastrointestinal and Liver Unit, Prince of Wales Hospital, University of New South Wales, Sydney, Australia.

Received: 11 December 2019 Accepted: 15 February 2020

Published online: 05 March 2020

\section{References}

1. Lastovica AJ, On SLW, Zhang L. The family Campylobacteraceae. In: Rosenberg E, DeLong EF, Lory S, Stackebrandt E, Thompson F, editors. The prokaryotes: deltaproteobacteria and epsilonproteobacteria. Berlin: Springer; 2014. p. 307-35.

2. Liu F, Ma R, Wang Y, Zhang L. The clinical importance of Campylobacter concisus and other human hosted Campylobacter species. Front Cell Infect Microbiol. 2018;8:243.

3. Hazards EPoB. Scientific opinion on quantification of the risk posed by broiler meat to human campylobacteriosis in the EU. EFSA J. 2010;8(1):1437.

4. Marder ME, Griffin PM, Cieslak PR, et al. Preliminary incidence and trends of infections with pathogens transmitted commonly through foodfoodborne diseases active surveillance network, 10 U.S. Sites, 2006-2017. Altanta: Centers for Disease Control and Prevention; 2018.
5. Sammarco ML, Ripabelli G, Fanelli I, Grasso GM, Tamburro M. Prevalence and biomolecular characterization of Campylobacter spp. isolated from retail meat. J Food Prot. 2010:73(4):720-8.

6. O'Leary MC, Harding O, Fisher L, Cowden J. A continuous commonsource outbreak of campylobacteriosis associated with changes to the preparation of chicken liver pâté. Epidemiol Infect. 2009;137(3):383-8.

7. Rahimi E, Ameri M, Kazemeini HR. Prevalence and antimicrobial resistance of Campylobacter species isolated from raw camel, beef, lamb, and goat meat in Iran. Foodborne Pathog Dis. 2010;7(4):443-7.

8. Graham C, Whyte R, Gilpin B, Cornelius A, Hudson JA, Morrison D, et al. Outbreak of campylobacteriosis following pre-cooked sausage consumption. Aust N Z J Public Health. 2005:29(6):507-10.

9. Miller WG, Parker CT, Heath S, Lastovica AJ. Identification of genomic differences between Campylobacter jejuni subsp. jejuni and C. jejuni subsp. doylei at the nap locus leads to the development of a C. jejuni subspeciation multiplex PCR method. BMC Microbiol. 2007;7(1):11.

10. Miller WGMR. Prevalence of Campylobacter in the food and water supply: incidence, outbreaks, isolation and detection. In: Konkel MEKJ, editor. Campylobacter: molecular and cellular biology. Norwich: Horizon Scientific Press; 2005. p. 101-63.

11. Zhang L, Budiman V, Day AS, Mitchell H, Lemberg DA, Riordan SM, et al. Isolation and detection of Campylobacter concisus from saliva of healthy individuals and patients with inflammatory bowel disease. J Clin Microbiol. 2010;48(8):2965-7.

12. Mahendran V, Riordan SM, Grimm MC, Tran TAT, Major J, Kaakoush NO, et al. Prevalence of Campylobacter species in adult Crohn's disease and the preferential colonization sites of Campylobacter species in the human intestine. PLOS ONE. 2011;6(9):e25417-e.

13. Mukhopadhya I, Thomson JM, Hansen R, Berry SH, El-Omar EM, Hold GL. Detection of Campylobacter concisus and other Campylobacter species in colonic biopsies from adults with ulcerative colitis. PLOS ONE. 2011;6(6):e21490

14. Man SM, Zhang L, Day AS, Leach ST, Lemberg DA, Mitchell H. Campylobacter concisus and other Campylobacter species in children with newly diagnosed Crohn's disease. Inflamm Bowel Dis. 2010;16(6):1008-16.

15. Liu F, Ma R, Tay CYA, Octavia S, Lan R, Chung HKL, et al. Genomic analysis of oral Campylobacter concisus strains identified a potential bacterial molecular marker associated with active Crohn's disease. Emerg Microbes Infect. 2018;7(1):64.

16. Kirk KF, Nielsen HL, Thorlacius-Ussing O, Nielsen H. Optimized cultivation of Campylobacter concisus from gut mucosal biopsies in inflammatory bowel disease. Gut Pathog. 2016:8(1):27.

17. Nielsen HL, Ejlertsen T, Engberg J, Nielsen $\mathrm{H}$. High incidence of Campylobacter concisus in gastroenteritis in North Jutland, Denmark: a population-based study. Clin Microbiol Infect. 2013;19(5):445-50.

18. Macfarlane S, Furrie E, Macfarlane GT, Dillon JF. Microbial colonization of the upper gastrointestinal tract in patients with Barrett's esophagus. Clin Infect Dis. 2007;45(1):29-38.

19. Man SM, Zhang L, Day AS, Leach ST, Lemberg DA, Mitchell H. Campylobacter concisus and other Campylobacter species in children with newly diagnosed Crohn's disease. Inflamm Bowel Dis. 2009;16(6):1008-16.

20. Vandamme P, De Ley J. Proposal for a new family, Campylobacteraceae. Int J Syst Bacteriol. 1991:41:451-5.

21. Miller WG. Comparative genomics of Campylobacter species other than Campylobacter jejuni. In: Campylobacter. 3rd edn. American Society of Microbiology; 2008

22. Vorwerk H, Huber C, Mohr J, Bunk B, Bhuju S, Wensel O, et al. A transferable plasticity region in Campylobacter coli allows isolates of an otherwise non-glycolytic food-borne pathogen to catabolize glucose. Mol Microbiol. 2015;98(5):809-30.

23. Muraoka WT, Zhang Q. Phenotypic and genotypic evidence for L-fucose utilization by Campylobacter jejuni. J Bacteriol. 2011;193(5):1065-75.

24. Stahl M, Friis LM, Nothaft H, Liu X, Li J, Szymanski CM, et al. L-Fucose utilization provides Campylobacter jejuni with a competitive advantage. Proc Natl Acad Sci. 2011;108(17):7194-9.

25. Hofreuter D. Defining the metabolic requirements for the growth and colonization capacity of Campylobacter jejuni. Front Cell Infect Microbiol. 2014:4:137.

26. Parkhill J, Wren BW, Mungall K, Ketley JM, Churcher C, Basham D, et al. The genome sequence of the food-borne pathogen Campylobacter jejuni reveals hypervariable sequences. Nature. 2000;403(6770):665-8. 
27. Pearson BM, Gaskin DJ, Segers RP, Wells JM, Nuijten PJ, van Vliet AH. The complete genome sequence of Campylobacter jejuni strain 81116 (NCTC11828). J Bacteriol. 2007;189(22):8402-3.

28. Guccione E, Del Rocio Leon-Kempis M, Pearson BM, Hitchin E, Mulholland F, Van Diemen PM, et al. Amino acid-dependent growth of Campylobacter jejuni: key roles for aspartase (AspA) under microaerobic and oxygen-limited conditions and identification of AspB (Cj0762), essential for growth on glutamate. Mol Microbiol. 2008;69(1):77-93.

29. Leach S, Harvey P, Wait R. Changes with growth rate in the membrane lipid composition of and amino acid utilization by continuous cultures of Campylobacter jejuni. J Appl Microbiol. 1997;82(5):631-40.

30. Line JE, Hiett KL, Guard-Bouldin J, Seal BS. Differential carbon source utilization by Campylobacter jejuni 11168 in response to growth temperature variation. J Microbiol Methods. 2010;80(2):198-202.

31. Westfall HN, Rollins DM, Weiss E. Substrate utilization by Campylobacter jejuni and Campylobacter coli. Appl Environ Microbiol. 1986;52(4):700-5.

32. Wright JA, Grant AJ, Hurd D, Harrison M, Guccione EJ, Kelly DJ, et al. Metabolite and transcriptome analysis of Campylobacter jejuni in vitro growth reveals a stationary-phase physiological switch. Microbiology. 2009:155(1):80-94.

33. Woodall CA, Jones MA, Barrow PA, Hinds J, Marsden GL, Kelly DJ, et al. Campylobacter jejuni gene expression in the chick cecum: evidence for adaptation to a low-oxygen environment. Infect Immun. 2005:73(8):5278-85.

34. van der Stel AX, Boogerd FC, Huynh S, Parker CT, van Dijk L, van Putten JPM, et al. Generation of the membrane potential and its impact on the motility, ATP production and growth in Campylobacter jejuni. Mol Microbiol. 2017;105(4):637-51.

35. Hoffman PS, Goodman TG. Respiratory physiology and energy conservation efficiency of Campylobacter jejuni. J Bacteriol. 1982;150(1):319-26.

36. Kelly DJ. Complexity and versatility in the physiology and metabolism of Campylobacter jejuni. In: Campylobacter. 3rd edn. American Society of Microbiology; 2008.

37. Pajaniappan M, Hall JE, Cawthraw SA, Newell DG, Gaynor EC, Fields JA, et al. A temperature-regulated Campylobacter jejuni gluconate dehydrogenase is involved in respiration-dependent energy conservation and chicken colonization. Mol Microbiol. 2008;68(2):474-91.

38. Vegge CS, Jansen van Rensburg MJ, Rasmussen JJ, Maiden MCJ, Johnsen LG, Danielsen M, et al. Glucose metabolism via the Entner-Doudoroff pathway in Campylobacter: a rare trait that enhances survival and promotes biofilm formation in some isolates. Front Microbiol. 2016;7:1877.

39. Thomas MT, Shepherd M, Poole RK, van Vliet AHM, Kelly DJ, Pearson BM. Two respiratory enzyme systems in Campylobacter jejuni NCTC 11168 contribute to growth on I-lactate. Environ Microbiol. 2011;13(1):48-61.

40. Mendz GL, Ball GE, Meek DJ. Pyruvate metabolism in Campylobacter spp. Biochim Biophys Acta Gen Subj. 1997;1334(2):291-302.

41. Weerakoon DR, Olson JW. The Campylobacter jejuni NADH: ubiquinone oxidoreductase (complex I) utilizes flavodoxin rather than NADH. J Bacteriol. 2008;190(3):915-25.

42. Weingarten RA, Taveirne ME, Olson JW. The dual-functioning fumarate reductase is the sole succinate:quinone reductase in Campylobacter jejuni and is required for full host colonization. J Bacteriol. 2009;191(16):5293-300.

43. Weerakoon DR, Borden NJ, Goodson CM, Grimes J, Olson JW. The role of respiratory donor enzymes in Campylobacter jejuni host colonization and physiology. Microb Pathog. 2009;47(1):8-15.

44. Myers JD, Kelly DJ. A sulphite respiration system in the chemoheterotrophic human pathogen Campylobacter jejuni. Microbiology. 2005;151(1):233-42.

45. Jackson RJ, Elvers KT, Lee LJ, Gidley MD, Wainwright LM, Lightfoot J, et al. Oxygen reactivity of both respiratory oxidases in Campylobacter jejuni: the $C y d A B$ genes encode a cyanide-resistant, low-affinity oxidase that is not of the cytochrome bd type. J Bacteriol. 2007;189(5):1604-15.

46. Guccione EJ, Kendall JJ, Hitchcock A, Garg N, White MA, Mulholland F, et al. Transcriptome and proteome dynamics in chemostat culture reveal how Campylobacter jejuni modulates metabolism, stress responses and virulence factors upon changes in oxygen availability. Environ Microbiol. 2017;19(10):4326-48.

47. Pittman MS, Kelly DJ. Electron transport through nitrate and nitrite reductases in Campylobacter jejuni. Biochem Soc Trans. 2005;33(Pt 1):190-2.
48. Pittman MS, Elvers KT, Lee L, Jones MA, Poole RK, Park SF, et al. Growth of Campylobacter jejuni on nitrate and nitrite: electron transport to NapA and NrfA via NrfH and distinct roles for NrfA and the globin Cgb in protection against nitrosative stress. Mol Microbiol. 2007;63(2):575-90.

49. Sellars MJ, Hall SJ, Kelly DJ. Growth of Campylobacter jejuni supported by respiration of fumarate, nitrate, nitrite, trimethylamine- $N$-oxide, or dimethyl sulfoxide requires oxygen. J Bacteriol. 2002;184(15):4187-96

50. Liu YW, Denkmann K, Kosciow K, Dahl C, Kelly DJ. Tetrathionate stimulated growth of Campylobacter jejuni identifies a new type of bi-functional tetrathionate reductase $(\mathrm{Tsd} A)$ that is widely distributed in bacteria. Mol Microbiol. 2013;88(1):173-88.

51. Benoit SL, Maier RJ. Site-directed mutagenesis of Campylobacter concisus respiratory genes provides insight into the pathogen's growth requirements. Sci Rep. 2018;8(1):14203.

52. Kirk KF, Méric G, Nielsen HL, Pascoe B, Sheppard SK, Thorlacius-Ussing O, et al. Molecular epidemiology and comparative genomics of Campylobacter concisus strains from saliva, faeces and gut mucosal biopsies in inflammatory bowel disease. Sci Rep. 2018;8(1):1902.

53. Day WA Jr, Sajecki JL, Pitts TM, Joens LA. Role of catalase in Campylobacter jejuni intracellular survival. Infect Immun. 2000;68(11):6337-45.

54. Flint A, Sun Y-Q, Butcher J, Stahl M, Huang H, Stintzi A. Phenotypic screening of a targeted mutant library reveals Campylobacter jejuni defenses against oxidative stress. Infect Immun. 2014;82(6):2266-75.

55. Tanner ACR, Badger S, Lai C-H, Listgarten MA, Visconti RA, Socransky SS. Wolinella gen. nov., Wolinella succinogenes (Vibrio succinogenes Wolin et al.) comb. nov., and Description of Bacteroides gracilis sp. nov., Wolinella recta sp. nov., Campylobacter concisus sp. nov., and Eikenella corrodens from humans with periodontal disease. Int J Syst Evol Microbiol. 1981:31(4):432-45.

56. Reaney SK, Begg C, Bungard SJ, Guest JR. Identification of the D-tartrate dehydratase genes (ttdA and ttdB) of Escherichia coli and evolutionary relationship with the class I fumarase genes. J Gen Microbiol. 1993;139(7):1523-30.

57. Smith I, Hong-Shum L. Food additives data book. Hoboken: Wiley; 2011.

58. Hofreuter D, Mohr J, Wensel O, Rademacher S, Schreiber K, Schomburg $\mathrm{D}$, et al. Contribution of amino acid catabolism to the tissue specific persistence of Campylobacter jejuni in a murine colonization model. PLoS ONE. 2012;7(11):e50699-e.

59. Velayudhan J, Kelly DJ. Analysis of gluconeogenic and anaplerotic enzymes in Campylobacter jejuni: an essential role for phosphoenolpyruvate carboxykinase. Microbiology. 2002;148(3):685-94.

60. Lee H, Ma R, Grimm MC, Riordan SM, Lan R, Zhong L, et al. Examination of the anaerobic growth of Campylobacter concisus strains. Int J Microbiol. 2014;2014:476047.

61. Ma R, Liu F, Yap SF, Lee H, Leong RW, Riordan SM, et al. The growth and protein expression of inflammatory bowel disease-associated Campylobacter concisus is affected by the derivatives of the food additive fumaric acid. Front Microbiol. 2018;9:896.

62. Axley MJ, Grahame DA, Stadtman TC. Escherichia coli formate-hydrogen lyase. Purification and properties of the selenium-dependent formate dehydrogenase component. J Biol Chem. 1990;265(30):18213-8.

63. Pesci EC, Cottle DL, Pickett CL. Genetic, enzymatic, and pathogenic studies of the iron superoxide dismutase of Campylobacter jejuni. Infect Immun. 1994;62(7):2687-94.

64. Ohsawa I, Ishikawa M, Takahashi K, Watanabe M, Nishimaki K, Yamagata K, et al. Hydrogen acts as a therapeutic antioxidant by selectively reducing cytotoxic oxygen radicals. Nat Med. 2007;13(6):688-94.

65. Kern M, Simon J. Electron transport chains and bioenergetics of respiratory nitrogen metabolism in Wolinella succinogenes and other Epsilonproteobacteria. Biochim Biophys Acta Bioenerg. 2009;1787(6):646-56.

66. Nambu T, Wang D, Mashimo C, Maruyama H, Kashiwagi K, Yoshikawa K, et al. Nitric oxide donor modulates a multispecies oral bacterial community —an in vitro study. Microorganisms. 2019;7(9):353.

67. Cornelius AJ, Miller WG, Lastovica AJ, On SLW, French NP, Vandenberg $\mathrm{O}$, et al. Complete genome sequence of Campylobacter concisus ATCC $33237(\mathrm{~T})$ and draft genome sequences for an additional eight well-characterized C. concisus strains. Genome Announc. 2017;5(29):e00711-17.

68. Altschul SF, Gish W, Miller W, Myers EW, Lipman DJ. Basic local alignment search tool. J Mol Biol. 1990;215(3):403-10. 
69. Pearson WR. An introduction to sequence similarity ("Homology") searching. Curr Protoc Bioinform. 2013:42(1):3.1-3.1.8

70. Sondergaard D, Pedersen CN, Greening C. HydDB: a web tool for hydrogenase classification and analysis. Sci Rep. 2016;6:34212.

71. Schoneich C. Methionine oxidation by reactive oxygen species: reaction mechanisms and relevance to Alzheimer's disease. Biochem Biophys Acta. 2005;1703(2):111-9.

72. Gundogdu O, Bentley SD, Holden MT, Parkhill J, Dorrell N, Wren BW. Re-annotation and re-analysis of the Campylobacter jejuni NCTC11168 genome sequence. BMC Genomics. 2007;8:162.
73. Kanehisa M, Goto S. KEGG: kyoto encyclopedia of genes and genomes. Nucleic Acids Res. 2000;28(1):27-30

\section{Publisher's Note}

Springer Nature remains neutral with regard to jurisdictional claims in published maps and institutional affiliations.
Ready to submit your research? Choose BMC and benefit from:

- fast, convenient online submission

- thorough peer review by experienced researchers in your field

- rapid publication on acceptance

- support for research data, including large and complex data types

- gold Open Access which fosters wider collaboration and increased citations

- maximum visibility for your research: over $100 \mathrm{M}$ website views per year

At BMC, research is always in progress.

Learn more biomedcentral.com/submissions 